\title{
Quasi-Quadrature Solution of Integral Equations Fredholm of the Second Kind in the Class of Integrable Functions
}

\author{
Igor Petrovich Dobrovolsky \\ Institute of Physics of the Earth, Russian Academy of Sciences, Moscow, Russia \\ Email: dipedip@gmail.com
}

How to cite this paper: Dobrovolsky, I.P. (2019) Quasi-Quadrature Solution of Integral Equations Fredholm of the Second Kind in the Class of Integrable Functions. Open Access Library Journal, 6: e5736. https://doi.org/10.4236/oalib.1105736

Received: August 26, 2019

Accepted: September 9, 2019

Published: September 12, 2019

Copyright $\odot 2019$ by author(s) and Open Access Library Inc.

This work is licensed under the Creative Commons Attribution International License (CC BY 4.0).

http://creativecommons.org/licenses/by/4.0/

\begin{abstract}
The analog of the quadrature solution of the equation of Fredholm of the second kind is considered. Fundamental difference from classical quadrature formulas is as follows. On segments of the chosen grid not values of functions, but their integral average values are used. Computing examples show expediency of such approach in appropriate cases.
\end{abstract}

\section{Subject Areas \\ Integral Equation}

Keywords

Integral Average Value

\section{Introduction}

Numerical methods of the solution of the equations are universal. This is their important quality. Quadrature formulas are quite often used in the solution of the integral equations. Quadrature formulas are applicable only to continuous functions. In other words, the integrable function has to have a certain value at each point. This requirement cannot be fulfilled for the class of integrable functions. In such situation the alternative version of the quadrature solution of the integral equation is possible. This work is further work [1].

\section{Algorithm}

The essence of the offered algorithm is as follows. On the main segment of the integral equation the system of segments is formed and on each such segment the integral average value of function is defined. 
The equation is considered

$$
y(x)-\int_{a}^{b} K(x, t) y(t) \mathrm{d} t=f(x)
$$

which has the unique solution in a class of the integrable functions.

We form on a segment $[a, b]$ a uniform grid of $N$ segments with a step

$$
h=\frac{b-a}{N}
$$

On this grid there is $N+1$ of points. We build segments $\delta_{i}$ in the vicinity of each point

$$
\begin{aligned}
& \delta_{0}=\left[a, a+\frac{h}{2}\right], \\
& \delta_{i}=\left[a+\frac{h}{2}(2 i-1), a+\frac{h}{2}(2 i+1)\right], i=1, \cdots, N-1, \\
& \delta_{N}=\left[b-\frac{h}{2}, b\right]
\end{aligned}
$$

We will enter concept of integral average values on these segments. They have the form for functions $y(x)$ and $f(x)$

$$
w_{i}=\frac{1}{\delta_{i}} \int_{\delta_{i}} y(x) \mathrm{d} x, \quad f_{j}=\frac{1}{\delta_{j}} \int_{\delta_{j}} f(x) \mathrm{d} x
$$

Let's provide the function $y(t)$ under integral in (2.1) in the form

$$
y(t)=\sum_{i=0}^{N} w_{i} \delta_{i}
$$

where $\delta_{i}$ is characteristic function of the corresponding segment.

Then (2.1) receives the form

$$
y(x)-\sum_{i=0}^{N} K_{i}(x) w_{i}=f(x)
$$

where $K_{i}(x)=\int_{\delta_{i}} K(x, t) \mathrm{d} t$.

Let's carry out to (2.6) integral averaging on segments $\delta_{j}$

$$
w_{j}-\sum_{i=1}^{N} K_{j i} w_{i}=f_{j}, j=0, \cdots, N
$$

where $K_{j i}=\frac{1}{\delta_{j}} \int_{\delta_{j}} \int_{\delta_{i}} K(x, t) \mathrm{d} t \mathrm{~d} x, f_{j}=\frac{1}{\delta_{j}} \int_{\delta_{j}} f(x) \mathrm{d} x, j$ is number of the equation, $i$ is number of item in the equation.

(2.7) is system of the equations for definition of integral average values of unknown function on system of segments (2.3).

\section{Example 1}

The equation is considered

$$
y(x)-\int_{0}^{1} \frac{y(t) \mathrm{d} t}{\sqrt{|t-x|}}=\sqrt{x}-\frac{x \pi}{2}+\frac{x \ln x}{2}-\sqrt{1-x}-\frac{1}{2} x \ln (2-x+2 \sqrt{1-x})
$$


which has the exact solution $y=\sqrt{x}$.

Results of the quasi-quadrature solution of this equation are presented in Table 1.

In Table $1 y_{0}$ and $y_{N}$ are the integral average values calculated according to the exact solution. It is possible to claim that the received solution is satisfactory.

\section{Example 2}

The equation is considered

$$
y(x)-\int_{0}^{1} \sqrt{|t-x|} y(t) \mathrm{d} t=\frac{1}{\sqrt{x}}-\frac{x \pi}{2}-\frac{x \ln x}{2}-\sqrt{1-x}+\frac{1}{2} x \ln (2-x+2 \sqrt{1-x})
$$

which has the exact solution $y=\frac{1}{\sqrt{x}}$.

Results of the quasiquadrature solution of this equation are presented in $\mathrm{Ta}$ ble 2.

Let's make some analysis of the received solution. We see that the function $y(x)$ grows at approach to a point $x=0$. We will assume that this growth has power character. Specifically: we will assume that in the vicinity of a point $x=0$ function has the form $\bar{y}=p+q x^{n}$ with unknown $p, q$ and $n$. Then

$$
\bar{y}_{0}=\frac{2}{h} \int_{0}^{h / 2} \bar{y} \mathrm{~d} x=p+\frac{q}{2^{n}(n+1) N^{n}}
$$

Table 1. Quasiquadrature solution of Equation (3.1).

\begin{tabular}{ccccc}
\hline \multirow{2}{*}{$N$} & \multicolumn{2}{c}{ segment $\delta_{0}$} & \multicolumn{2}{c}{ segment $\delta_{N}$} \\
\cline { 2 - 5 } & $W_{0}$ & $y_{0}$ & $w_{N}$ & $y_{N}$ \\
\hline 20 & 0.11104 & 0.10541 & 0.99347 & 0.99372 \\
40 & 0.07689 & 0.07454 & 0.99647 & 0.99686 \\
80 & 0.05372 & 0.05270 & 0.99825 & 0.99843 \\
160 & 0.03773 & 0.03727 & 0.99914 & 0.99921 \\
320 & 0.02657 & 0.02635 & 0.99958 & 0.99960 \\
640 & 0.01871 & 0.01863 & 0.99976 & 0.99980 \\
\hline
\end{tabular}

Table 2. Quasiquadrature solution of Equation (4.1).

\begin{tabular}{ccccc}
\hline \multirow{2}{*}{$N$} & \multicolumn{2}{c}{ segment $\delta_{0}$} & \multicolumn{2}{c}{ segment $\delta_{N}$} \\
\cline { 2 - 5 } & $W_{0}$ & $y_{0}$ & $W_{N}$ & $y_{N}$ \\
\hline 20 & 12.647499 & 12.649110 & 1.00249 & 1.00632 \\
40 & 17.888020 & 17.888543 & 1.00166 & 1.00314 \\
80 & 25.298077 & 25.298221 & 1.00100 & 1.00156 \\
160 & 35.777063 & 35.777088 & 1.00057 & 1.00078 \\
240 & 43.817803 & 43.817805 & 1.00041 & 1.00052 \\
320 & 50.596448 & 50.596443 & 1.00031 & 1.00039 \\
\hline
\end{tabular}


For definition of unknown $p, q$ and $n$ we equate the received expression to three values from Table 2

$$
\begin{aligned}
& p+\frac{q}{2^{n}(n+1) 160^{n}}=w_{0}(160)=35.777063 \\
& p+\frac{q}{2^{n}(n+1) 240^{n}}=w_{0}(240)=43.817803 \\
& p+\frac{q}{2^{n}(n+1) 320^{n}}=w_{0}(320)=50.596448
\end{aligned}
$$

The solution of system (4.3) gives (after rounding) $n=-0.5, p=0, q=1$. This solution correctly defines behavior of function $y(x)$.

\section{Conclusion}

The conducted research shows that the method of the quasi-quadrature solution can take its place among other methods of approximate solution of integral equations.

\section{Conflicts of Interest}

The author declares no conflicts of interest regarding the publication of this paper.

\section{References}

[1] Dobrovolsky, I.P. (2015) The Estimation of the Error at Richardson's Extrapolation and the Numerical Solution of Integral Equations of the Second Kind. Open Access Library Journal, 2, e2051. http://dx.doi.org/10.4236/oalib.1102051 